\title{
Modernising sexual health and HIV services in the UK
}

A n ordinary general meeting of the British Association for Sexual Health and HIV (BASHH) was held on 14 January 2005. The main theme of the meeting was to promote modernisation and service innovation in sexual health and HIV services. The meeting was co-hosted by the BASHH Education and Clinical Governance committees.

In his keynote address, Dr Immy Ahmed-Jushuf emphasised that modernisation was not a new concept within the specialty-rather an evolving theme which has been evident over the past 20 years and he outlined some of the external as well as internal drivers for change. Sexual health services still face major challenges because of unprecedented demand, resulting in unacceptable waiting time for patients wishing to access the service at a time when there has been a significant increase in the incidence of sexually transmitted infections (STI) and HIV. Capacity for seeing new attendees in the service had increased by 36.5\% between 1990 and 2002, and HIV workload has doubled between 1999 and 2002. There has been very little new investment in the service over the past 10 years. This significant increase in capacity has therefore been achieved through a range of initiatives including the introduction of new ways of working, revising the roles of nonmedical clinicians, and a reduction in follow up cases. Over the past 4 years, HIV testing uptake within GUM clinics has increased from $26 \%$ in 2001 to $67 \%$ in 2003-well in advance of the targets set in the National Strategy for Sexual Health and HIV for England.

Dr Ahmed argued that while there was undisputed need for more resources, the time was also right for GUM clinicians to systemically review their policies underpinning the service and the roles of healthcare professionals delivering the service.

Six presentations were made-four of which focused on innovations that led to enhancing service capacity, while two focused in improving service quality.

Vanessa Griffiths, on behalf of the 12 centres that participated in the SixSigma project, outlined the use of this management tool to systematically audit the reasons for follow up visits with a view to significantly reduce these, thus increasing capacity for new patients. The group was well on target to reduce the follow up:new ratio to 0.5 (current national average 1.3).

Dr Alan McOwan from the Chelsea and Westminster Hospital, London, described Option E, a pilot project where appropriately trained HIV nurse specialists offered follow up clinics for patients who were stable on antiretroviral treatment (HAART). The nurses worked within agreed protocols. Patients were asked to choose the method of receiving medication-either by calling in at the clinic or having medications delivered to their preferred address. A prescribing pharmacist or doctor reviewed results, which were then emailed to the patient, together with details of drug delivery/ collection. Patients opting for this service would be offered a formal medical review annually.

The results of 5 months of the pilot were presented. They showed high levels of patient satisfaction and significant savings in pharmacy costs for the 29 patients who were enrolled on the programme. The potential for enhancing medical staff capacity was also highlighted.

The advent of non-invasive STI diagnostic tests has opened up new possibilities for "fast track" screening of asymptomatic individuals and greater choice for service users.

Dr Steve Baguley from Aberdeen described a project that offered asymptomatic women and heterosexual men over the age of 17 the option for "rapid screening." Those found suitable for this service were asked to provide a specimen (first catch urine for men and self obtained lower vaginal swab for women), which was tested for chlamydia using a nucleic acid amplification test. Those who tested positive were recalled and offered full STI screening and treatment. This service has reported high levels of user satisfaction.

Carol Bailey from the Manchester Centre for Sexual Health and the Palatine Centre, Manchester, described how patients attending the family planning services in Manchester diagnosed with uncomplicated STIs were offered full STI screening, treatment, partner notification, and treatment with the family planning service by a specialist nurse, thus reducing pressure on GUM services. Over a 9 month period, 245 patients were treated according to national guidelines, and contact tracing rates comparable to those seen in GUM clinics were achieved.

Dr Hugo McLean from Hull described the use of an oral transudate test for screening for HIV, syphilis, and hepatitis $B$ and $C$ in patients who reported needle phobia or those who declined venepuncture for other reasons.

Louise Fellows from the Whittal Street Clinic, Birmingham described the introduction of a new patient registration document, which clearly explained to all new patients the level of confidentiality that the service offered. An explicit statement outlining how their data would be used and under what circumstances it could be shared with those not directly involved in their care was provided. Patients were asked to sign their acceptance of the "contract." This was seen as a more effective way of informing service users their rights and clarifying the limits to confidentiality.

Dr Rak Nandwani paid tribute to all the presenters and hoped that the meeting would provide food for thought to other colleagues who might wish to introduce similar initiatives. He stressed the importance for BASHH to provide forums where innovations and good practice could be shared, but warned against introducing change without appropriate evaluation.

I H Ahmed-Jushuf Department of Genitourinary Medicine Nottingham City Hospital, Hucknall Road,
Nottingham NG5 IPB UK. Nottingham NG5 1PB, UK;
iahmed@ncht.trent.nhs.uk

R Nandwani

Genitourinary Medicine Services, The Sandyford Initiative, 2-6 Sandyford Place, Glasgow G3 7MB, UK

M A Kingston

Department of Genitourinary Medicine Manchester Royal Infirmary, Oxford Road, Manchester M3 9WL, UK

M Abbott

Department of Genitourinary Medicine, Southport and Formby District General Hospital, Town Lane, Kew, Southport PR8 6PM 